\title{
Physiopathology of human embryonic implantation: clinical incidences
}

\author{
Philippe Merviel ${ }^{1}$, Emmanuelle Lourdel ${ }^{1}$, Rosalie Cabry ${ }^{2}$, Véronique Boulard ${ }^{2}$, \\ Mélanie Brzakowski ${ }^{1}$, Pauline Demailly ${ }^{2}$, Françoise Brasseur ${ }^{2}$, Henri Copin ${ }^{2}$, \\ Aviva Devaux ${ }^{2}$
}

${ }^{1}$ Obstetric, Gynecologic and ART department, University hospital Amiens, 124 rue Camille Desmoulins, 80054 Amiens cedex 1 - France

${ }^{2}$ Cytogenetic and Reproductive Biology department, University hospital Amiens, 124 rue Camille

De moulins, 80054 Amiens cedex 1 - France

\begin{abstract}
Embryo implantation consists of a series of events promoting the invasion of the endometrium and then the uterine arterial system by the extra-embryonic trophoblast. In order for this semi-heterologous implantation to succeed, the endometrium has to first undergo a number of structural and biochemical changes (decidualization). The decidua's various constituents subsequently play a role in the embryonic implantation. The third step is the transformation of the uterine vascular system and the growth of the placenta, which will provide the foetoplacental unit with nutrients. Several physiopathological aspects will be discussed: 1) the implantation window, regulated by maternal and embryonic hormonal secretions and thus influenced by any defects in the latter: dysharmonic luteal phase, 21-hydroxylase block, abnormal integrin expression, 2) the successive trophoblast invasions of uterine vessels which, when defective, lead to early embryo loss or late-onset vascular pathologies, as preeclampsia, 3) the pregnancy's immunological equilibrium, with a spontaneously tolerated semi-allogeneic implant, 4) the impact of pro-coagulant factors (thrombophilia) on the pregnancy's progression, 5) the environment of the uterus, ranging from hydrosalpinx to uterine contractions. In summary, the least anatomical or physiological perturbation can interfere with human embryonic implantation - a very particular phenomenon and a true biological paradox.
\end{abstract}

Keywords: endometrium, implantation, preeclampsia, progesterone, trophoblast

\section{Introduction}

Implantation of the human embryo leads to invasion of the endometrium by the extra-embryonic trophoblast and colonization of the uterine arteries. This phenomenon enables the embryo to anchor itself onto the uterine wall and thus maintenance of the pregnancy and foetal growth via the placenta. For this semi-allogenic graft to occur, the endometrium must first undergo a number of structural and biochemical modifications which are collectively referred to as decidualization. Hence, an "implantation window" opens as a result of the synchronization between embryonic development and endometrial maturity. Lastly, trophoblast cells

Correspondence: P. Merviel, University hospital Amiens, 124 rue Camille Desmoulins, 80054 Amiens cedex 1 - France ; tel.: (+333) 22533662, fax.: (+333) 22533690, e-mail:

Merviel.Philippe@chu-amiens.fr transform the uterine vascular system - a change which (together with the action of vasomotor factors) enables adequate nourishment of the foetoplacental unit.

\section{The physiopathology of human embryo implantation}

The extravillous cytotrophoblasts (EVCTs) must invade the decidua before being able to modify the walls of the spiral arteries. However, unlike tumour invasion or an inflammatory reaction, implantation is an invasion that is controlled in time and space. Any anomaly in invasion-promoting and invasion-limiting factors may cause a pregnancy-related disease, such as preeclampsia. In order to invade the decidua, the trophoblast cells need both to recognize (via integrins and cadherins) the various components of the membrane and the extracellular matrix (ECM) and then break 
them down (with metalloproteases). To control this invasion, the endometrium modifies the composition of the ECM and secretes transforming growth factor (TGF) and tissue metalloprotease inhibitors (TIMPs). Moreover, the decidua is colonized by immune system cells (NK cells, lymphocytes and macrophages) that are responsible for the local production of cytokines which promote or inhibit invasion by trophoblasts.

\section{Endometrial decidualization}

For the human embryo, implantation occurs when the endometrial tissue and vascular configurations have reached a receptive stage during the proliferative phase and the beginning of the luteal phase, under the influence of sex hormones, growth factors, angiogenic and vasomotor agents [1]. Thickening of the endometrium depends on the secretion of oestrogens during the follicular phase and is necessary but not sufficient for embryo implantation. Ideally, a thickness of 8 to 12 $\mathrm{mm}$ between the two outermost endometrial leaflets is required in the peri-ovulatory period. If endometrial atrophy $(<5 \mathrm{~mm})$ is observed on the $10^{\text {th }}$ day of the cycle, adjuvant treatment can legitimately be prescribed. The vaginal administration of micronized oestradiol has been suggested by Tourgeman et al. [2], since the endometrial thickness markedly increased following the prescription of vaginally administered oestradiol. Nitric oxide donors have also been tested, with varying results: Battaglia et al. [3] did not observe any effect of L-arginine on endometrial thickness, whereas Sher and Fisch's self-controlled study [4] of 4 female patients taking Viagra ${ }^{\circledR}$ showed significant increases in uterine vasodilation and endometrial thickness. This result shows nicely that even though the endometrium clearly depends on steroid hormones, better vascularization can also improve defects in endometrial thickness; this observation also explains why some mucosae remain thin, even when exposed to high oestradiol levels.

Except during pregnancy, the ECM is composed of collagens I, III, V and VI, fibronectin and periglandular tenascin deposits. During decidualization, the endometrial stromal (decidual) cells produce a pericellular matrix composed of collagen IV, laminin and heparan sulphate. Substantial hydration of the stroma occurs at the same time. These changes in the composition and hydration of the ECM make it easier for the EVCTs to invade the decidua. The modified ECM also establishes close contacts with the lymphoid cells present in the decidua, thereby increasing the cellular interactions between trophoblast and lymphoid cells. Decidual hydration is revealed by ultrasonography during the peri-ovulatory period, when one observes a triple-line endometrium with hypoechogenicity (oedema) on both sides of the central line of the uterine space. After ovulation, luteal secretion of progesterone will prompt the endometrium to become progressively hypoechogenic as it goes towards the uterine cavity. Fanchin et al. [5] studied this phenomenon by digitizing ultrasound scans and analyzing the endometrium's grey-scale intensity. Six hypoechogenic groups were defined: $\leq 30 \%, 31-40 \%, 41-50 \%, 51-60 \%, 61-70 \%$ and $>70 \%$, with pregnancy rates of $59 \%, 57 \%, 35 \%$, $20 \%, 16 \%$ and $11 \%$, respectively $(\mathrm{p}<0.001)$. It is noteworthy that on the day of the hCG, there were no intergroup differences in terms of endometrial thickness or oestradiol and progesterone levels. Fanchin thus concluded that early luteal differentiation of the endometrium (i.e. appearance of hyperechogenicity) is detrimental to implantation and thus recommended that embryos should be frozen (for transfer during a more appropriate cycle) or that an antiprogesterone drug (RU 486) should be used to delay progesteronedriven transformation of the endometrium.

In cases of pre-ovulatory progesterone secretion, the endometrium will display early maturation, which is unfavourable for embryo implantation. Hence, in collaboration with Professor Philippe Bouchard, we studied 15 women in an IVF programme (with a long agonist protocol) and who presented an abnormally high progesterone levels $(>2 \mathrm{ng} / \mathrm{ml})$ at the end of the follicular phase. This anomaly has been linked to a mutation in the gene for 21-hydroxylase (21-OHase), an enzyme involved in cortisol synthesis. A 21-OHase deficiency induces an increase in the syntheses of progesterone and 17-hydroxyprogesterone (17-OH-P) by the adrenal glands. The 21-OHase blockade was confirmed by assaying for 17-OH-P at baseline and after synacthen injection (ACTH). Endometrial biopsy samples from these women showed that endometrial maturation was occurring too early (according to the criteria of Noyes et al. [6]), as confirmed by the presence of glandular and stromal progesterone receptors at the end of the follicular phase. Hydrocortisone treatment enabled these women to achieve follicular-phase progesterone rates below $1 \mathrm{ng} / \mathrm{ml}$, re-establish normal endometrial maturation and obtain pregnancies.

This endometrial maturation is also associated with growth of the spiral arteries (branches of the uterine arteries), which will then carry maternal blood toward the intervillous spaces of the placenta. The growth rate and structure of the spiral arteries depend on ovarian hormonal secretions. Under the influence of oestrogens, the spiral arteries' diameter increases as they grow longer and become progressively twisted. This endothelial proliferation continues during the luteal phase and the first few weeks of gestation. Growth factors also play a role in neoangiogenesis: levels of fibroblast growth factor $\mathrm{b}$ (FGFb, a powerful angiogenic factor) are increased by oestradiol and inhibited by progesterone, vascular endothelial growth factor 
(VEGF, stimulated by oestrogens and hypoxia) is mitogenic for endothelial cells and increases vascular permeability, whereas platelet-derived growth factor (PDGF) contributes to angiogenesis and to the growth of smooth muscle cells. This neoangiogenesis can be disrupted by disease states that are associated with microangiopathy, such as insulin-dependent or gestational diabetes and chronic or gestational hypertension after a kidney transplant.

\section{Endometrium-embryo interactions}

The embryo (morula) arrives in the uterine cavity between 132 and 144 hours after fecundation. As soon as the trophectoderm is formed, it expresses LIF receptors; LIF is produced by the endometrium in the periimplantation period and is involved in differentiation of the cytotrophoblast into the syncytiotrophoblast, hCG secretion and the expression of cell surface integrins. The blastocyst secretes several factors, including hCG (stimulating the ovarian production of progesterone) and IL-1. Receptors for the latter are present at the surface of endometrial epithelial cells during the luteal phase and their activation leads to secretion of LIF; this clearly shows that a mother-embryo dialogue (driven by growth factors and cytokines) becomes established well before contact between the embryo and the endometrium [7].

Contact between the blastocytic trophoblast and the endometrium is necessarily preceded by blastocyst hatching, which is under the influence of the endometrial lysine production resulting from decidualisation. If hatching does not occur spontaneously, we can perform it mechanically or chemically as part of an IVF procedure.

Adhesion by EVCTs involves plasma membrane receptors (integrins and cadherins) that enable the cells to identify and then bind to ECM components (collagen IV, laminin, proteoglycans, heparan sulphate, entactin and fibronectin). Integrins are heterodimeric glycoproteins with 2 subunits $(\alpha$ and $\beta$ ), the combination of which forms many different integrins that bind to various ECM components [8]. The integrin subunit $\beta 3$ appears at day 20 of the cycle and coexists with subunits $\alpha 1, \alpha 4$ and $\alpha v$. Subunit $\alpha v \beta 3$ is thus an excellent marker for the implantation window. Loop interactions enable anchorage of the embryo: trophoblast $\alpha v \beta 3$ recognizes endometrial osteopontin and endometrial $\alpha v \beta 3$ interacts with the vitronectin and fibronectin expressed by the trophoblast (Fig. 1). Lessey et al. [9] has estimated that approximately two thirds of women with idiopathic infertility and all of those with delayed endometrial maturation have an $\alpha v \beta 3$ deficiency. When these women were treated with progesterone, histological findings for 16 of 23 patients returned to normal, with
ENDOMETRIUM

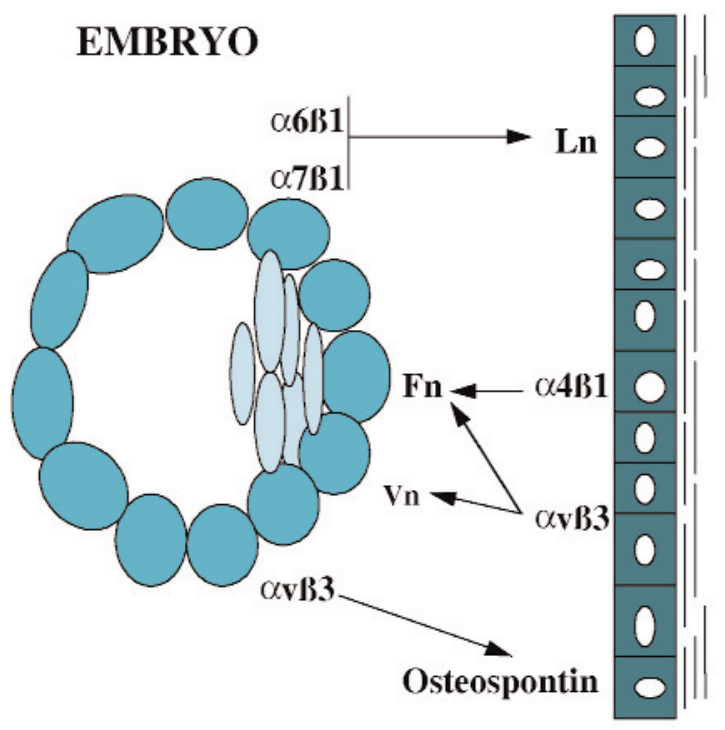

Fig. 1. Trophectoderm-Endometrium integrin interactions during implantation. Ln - laminin; Vn - vitronectin; Fn - fibronectin

11 out of 16 having an adequate $\beta 3$ level and 5 out of $16 \beta 3$-deficient women also displaying endometriosis. Creus et al. [10] performed two endometrial biopsies for each of 55 infertile women, one in the middle of the luteal phase (6 to 8 days after ovulation) and the other at the end (10-12 days). Nine patients had a defective, out-of-phase endometrium (i.e. a defective luteal phase, DLP) at the first biopsy but none were out of phase at the second biopsy. The only integrin-related difference between the DLP and the normal luteal phase (NLP) was that only the NLP group expressed integrin $\alpha v \beta 3$ in the first biopsy. We have studied plasma progesterone levels during these defective luteal phases (Merviel P. and Zorn JR, unpublished). A defective luteal phase (DLP) is characterized by a normal duration of elevated basal temperature ( $>11$ days), a normal plasma progesterone level at day $22(>5$ $\mathrm{ng} / \mathrm{ml}$ ) and an abnormal endometrial biopsy at day 22 (with a delay in endometrial maturation of approximately 2 days). We initiated hormone assays at the time of the LH surge initiating rise (LH-SIR) when LH plasma levels reach $180 \%$ of the baseline value. We observed that progesterone secretion was delayed from the peri-ovulatory period onwards (until 120 hours after the LH-SIR). When the plasma progesterone curves in the DLP and NLP were compared, a lag of approximately two days was observed. Early progesterone supplementation resulted in a normal endometrial biopsy at day 22. More generally, there was a clear $\alpha v \beta 3$ deficiency not only in patients with endometriosis or idiopathic infertility but also in those with hydrosalpinx, early miscarriage or polycystic ovary syndrome. 


\section{Trophoblast invasion of the decidua}

A "switch" modifies the integrin profile as the cytotrophoblast cells change from a proliferative state (at the base of anchoring villi) to an interstitial position (in the deepest portion of the decidua and spiral arteries) [11]. Thus, proliferative EVCTs first express only integrin $\alpha 6 \beta 4$ (a receptor for laminin, a component of the basement membrane) and then acquire the ability to express integrin $\alpha 5 \beta 1$ (a fibronectin receptor) as they become invasive and migrate. Once the cytotrophoblast cells have become interstitial and perivascular, integrin $\alpha 1 \beta 1$ (laminin and type I/IV collagen receptors) also appear.

Since the ECM is organized into a three-dimensional network that prevents passive cell migration, adhesion of trophoblast cells to ECM components is necessary but not sufficient for invasion. Accordingly, the trophoblast cell must be able to proteolyse the ECM components. Matrix metalloproteases (MMPs) are endoproteinases that require the presence of $\mathrm{Ca}^{++}$and $\mathrm{Zn}^{++}$ions and comprise 13 members in three families: collagenases (MMPs 1, 8, 13), which break down type I and III collagens; gelatinases A (MMP 2) and B (MMP 9), which break down gelatin, collagen IV and elastin; and the stromelysins (MMP 3, 7, 10 and 11), which have a broader spectrum. These enzymes are regulated by their level of activation (most are secreted as inactive zymogens) and the presence of TIMPs (secreted by the decidua) which block the enzyme's active site. The trophoblast cells secrete a number of MMPs during the first trimester, including MMP 9, which plays a primordial role in the invasion. Expression of TIMP 1 (which is highest at term) balances MMP 9 activity and thereby controls the invasiveness of the trophoblast. Furthermore, integrins can modulate MMP expression.

Tissue growth factor $\beta$ (TGF $\beta$ ) is expressed at the foetomaternal interface from the first trimester through to term and inhibits trophoblast proliferation and invasion; TGF $\beta 1$ and TGF $\beta 2$ are essentially expressed by the villi and the decidua, respectively. Tissue growth factor $\beta 1$ promotes formation of ECM (collagen and fibronectin, in particular), induces TIMP 1 expression and reduces EVCT migration by overexpressing $\alpha 5 \beta 1$. This overexpression makes the EVCTs adhere more strongly to the ECM and activates the differentiation of the cytotrophoblast into a non-invasive syncytiotrophoblast.

\section{Invasion of the uterine spiral arteries by the extra-villous cytotrophoblast}

The establishment of the uteroplacental vascular system begins with the invasion of the maternal decidua by EVCTs. Two successive, interdependent phenomena are needed to accomplish the complete transformation of the uterine spiral arteries by trophoblast cells [12].

\section{The first vascular invasion by the cytotrophoblast}

During the first trimester (from 5 to 8 weeks, approximately), the EVCTs sheath the outer wall of the decidual capillaries and the intra-endometrial branches of the uterine spiral arteries and thereby create a trophoblastic shell around these vessels. The trophoblast cells move from the exterior towards the inner capillary walls, where they become organized into loose but interlinked clusters within the trophoblastic shell. These intravascular plugs obstruct almost all of the decidual capillaries but act as a filter rather than a barrier; the permeability of these plugs enables plasma (and some maternal red blood cells) to diffuse towards pools of blood that have formed as a result of vascular invasion and which correspond to the future intervillous spaces [13].

This anatomical phenomenon results in an increase in oxygen partial pressure $\left(\mathrm{PO}_{2}\right)$ upstream of the plugs and a decrease downstream of them. The higher upstream $\mathrm{PO}_{2}$ diminishes lipid peroxidation in the endothelial cells of the intramyometrial spiral arteries, which in turn translates into an increase in levels of prostacyclin (the so-called prostaglandin $\mathrm{I}_{2}, \mathrm{PGI}_{2}$ ), a decrease in thromboxane $\mathrm{A}_{2}$ (TXA ${ }_{2}$ ) and a corresponding increase in local vasodilatation. The increase in $\mathrm{PO}_{2}$ also decreases production of the vasoconstrictor endothelin 1 (ET-1). Downstream of these plugs, the lower $\mathrm{PO}_{2}$ helps ensure the best possible environment for embryonic organogenesis. Conversely, the high pressure upstream of the plugs increases ET-induced nitric oxide (NO) release in the myometrial spiral arteries and thus helps to further increase local vasodilatation $[14,15]$. The placental $\mathrm{PO}_{2}$ (while lower than endometrial $\mathrm{PO}_{2}$ during the first trimester) increases progressively between 8 and 12 weeks. Moreover, since first-trimester embryos lack defence systems against oxygen free radicals, this low $\mathrm{PO}_{2}$ level protects their tissues against the harmful effects of oxygen [16]. Lastly, embryos at this term contain embryonic haemoglobin, which has a greater affinity for oxygen in low partial pressure conditions (such as those encountered in plasma).

The existence of plugs in the endometrial capillaries should theoretically be accompanied by a stacking of maternal red blood cells upstream and the appearance of extensive thrombi. However, this is not observed in vivo because the local factors that regulate local haemostasis (thrombomodulin, tissue factor and plasminogen activator) work together to (i) ensure that blood flows through the uterine spiral arteries and (ii) prevent extravasation following EVCT invasion. Thrombomodulin (TM) is secreted by the endothelial cells and activates protein $\mathrm{C}$, which has a proteolytic activity and inhibits the formation of blood clots. Via its anticoagulant action, TM prevents the formation of 
intravascular thromboses. Pro-coagulant tissue factor (TF) is a located on the membranes of the endometrial stromal cells (during the secretory phase) and the perivascular decidual cells. Secretion of TF can be secreted by progesterone and contributes to the perivascular, endometrial haemostasis required after EVCT vascular invasion by synthesizing thrombin (which transforms fibrinogen into fibrin). At the same time, fibrinolysis in the decidua is inhibited via activation of plasminogen activator inhibitor type 1 (PAI-1) and a decrease in tissue-type and urokinase-type plasminogen activators (tPA, uPA).

The plugs in the decidual capillaries provide haemodynamic protection for the embryo by preventing high vascular pressure in the blood lakes. The increased sinuosity of the spiral arteries at the beginning of pregnancy (which has a damper effect on maternal blood flow) and the extraembryonic coelom also play a role in this protection. In cases of spontaneous abortions, it is frequently observed that in the absence of intravascular plugs, the mother's blood has flooded the intervillous lakes [17]. At the beginning of pregnancy, the embryo evacuates its waste towards the yolk sac. There is no embryonic circulation and the pressure in the villous capillaries is lower than in the blood lakes. The transfer of nutrients and oxygen therefore occurs solely from the mother to the embryo. At 4-5 weeks, foetal heart activity begins and pressure in the villous capillaries increases thenceforth, enabling transfers from the embryo to the mother. The plugs in the maternal vessels protect these embryonic-maternal exchanges, since a substantial increase in the blood lake pressure would stop the transfers, cause collapse of the villous vessels and prevent the embryo from thriving. This would lead to cessation of embryonic-placental circulation and thus death of the embryo [18].

\section{The second vascular invasion by the cytotrophoblast}

Plugs in the intra-endometrial arteries can be observed from 5 weeks onwards and then progressively disaggregate from week 8 through to week 13 . The second and final trophoblast invasion of the intramyometrial spiral arteries thus occurs between 13 weeks and 18 weeks. Since these are continuous phenomena, intramyometrial vascular invasion can sometimes be observed before 13 weeks. A proportion of the trophoblast cells in the plugs can move backwards to colonize the surface of the inner wall of the intramyometrial spiral arteries and then penetrate deep into the vascular tissue [19]. This intraparietal encroachment causes the endothelial cells and smooth muscle cells of the tunica media and the internal elastic layer to disappear progressively [20]. The latter is replaced by a fib- rin deposit that deprives the vessels of their contractility. The trophoblast cells progressively develop an endothelial phenotype because of a switch from E- to VE-cadherin and the acquisition of endothelial cell molecules (such as VCAM-1 and PECAM-1) [21]. Decreased resistance in the uterine arteries triggers the continuous blood flow through the intervillous spaces that is required for foetal growth during the second and third trimesters. Intervillous blood flow increases at around 10-12 weeks of gestation and results in exposure of the trophoblast to higher oxygen tensions [22].

These anatomical and haemodynamic processes can be visualized in a Doppler study by the disappearance of the uterine artery notch, increased diastolic flow through these arteries and the blood flow that appears in the intervillous spaces. This progressive replacement of the collagen and elastin framework and the transformation of the intramyometrial spiral arteries is generally complete at 18 weeks but sometimes requires several additional weeks (it is not unusual for notches to disappear on Doppler ultrasound examinations between week 22 and week 26). During this period, the foetus acquires foetal haemoglobin $(\mathrm{HbF})$, the oxygen-uptake capacities of which match the greater needs for foetal growth.

Hence, there is a clear paternal role in the phenomenon of implantation (as in molar pregnancies, where paternal uniparental disomy is observed). In order to assess the influence of the decidual environment on placentation and trophoblast differentiation, we have collaborated with Professor J.M. Foidard to compare ectopic (tubal) pregnancies occurring in the absence of differentiated decidua with gestational age-matched normal pregnancies [23]. Differentiation of EVCTs in anchoring villi from both uterine and ectopic pregnancies showed a comparable switch from a proliferative to an invasive phenotype. Whereas $\alpha 6 \beta 4$ integrin, Ecadherin, EGF receptor and connexin 40 were immunolocalized in the proximal proliferative cells of the columns, $\alpha 5 \beta 1, \alpha 1 \beta 1, \mathrm{C}$ erb B2 and connexin 32 were expressed by invasive cytotrophoblasts. This immunohistochemical characterization of trophoblast differentiation suggests that the maternal decidua does not seem to be required for acquisition of the invasive phenotype during the first trimester of pregnancy.

Maternal red blood cells accumulate upstream of the plug and can thus form a clot in hypercoagulant states, such as when thrombophilia is present [24]. This clot blocks the passage of plasma ultrafiltrate and leads to embryonic failure. If a clot has formed upstream of the plugs, it will persist after plug disaggregation - leading to permanent blockage of the placental vascular bed and prevention of the trophoblasts' counter-current arterial migration. The absence of this second trophoblast invasion leads to pregnancy-associated vascular pathologies, such as preeclampsia 
$[25,26]$. The occurrence of recurrent miscarriages is thus associated with a higher incidence of pregnancyassociated vascular complications.

\section{Vasomotor factors}

Pregnancy is associated with a decrease in blood pressure, a drop in systemic and uterine vascular resistance and a reduced response to various vasopressor (vasoconstrictive) agents. The tone of the uteroplacental vessels is regulated by a variety of factors. Schematically, uterine vascular tone is mainly regulated by a vasoconstrictor system (endothelin/enkephalinase) and an opposing vasodilator system (nitric oxide/guanylate cyclase). These agents are involved in the placental regulation of vascular flow and set up a local balance that enables adequate blood intake [27]. Certain diseases (such as the vascular complications of hypertension) may be due to deregulation of these systems. It is now generally agreed that endothelial cells and endocrine \& nervous system factors are involved in the regulation of vasomotor tone and also prevent platelet aggregation.

Endothelin (ET) is one of the most powerful vasoconstrictive factors in the foetal-placental circulation. It is a 21-amino-acid peptide that exists in three isoforms (ET-1, ET-2 and ET-3) and which targets the spiral artery endothelial cells. Endothelin is broken down by the enzyme enkephalinase, the production of which is induced by progesterone. Thus, in the middle of the luteal phase (at the progesterone peak), the ET to enkephalinase ratio favours ET breakdown and therefore contributes to the absence of vasoconstriction of the implantation site vessels. Moreover, the hypothesis in which ET-1 is involved in disorders such as intrauterine growth retardation and preeclampsia seems to be confirmed by the elevated concentration of immunoreactive ET in both the umbilical vessels and the maternal blood in such cases and increased ET-1 gene expression in the placental villi in preeclampsia $[28,29]$.

When intact, endothelium produces a highly labile vasodilator product - nitric oxide (NO) - and triggers up-regulation of the local blood flow [15,30]. This gas (which can diffuse freely through cell membranes) penetrates the smooth muscle cells surrounding the vessels and relaxes them by activating cGMP [26]. Pregnancy is associated with increased NO production. Moncada et al. [31] showed that administration of inhibitors of NO synthase or guanylate cyclase amplifies the vasoconstriction observed with ET-1 and $\mathrm{TXA}_{2}$.

Oestrogens (in particular oestradiol) have both direct (i.e. non-genomic) and indirect (via protein synthesis) vasodilatory effects on the uterine arteries (via ET-1, NO and prostaglandins in the latter case) [32]. In fact, oestrogens facilitate $\mathrm{Ca}^{++}$entry into the endothelial cell, which leads to NO synthesis via stimulation of NO synthase and then to a relaxant effect on the muscle via cGMP. Endothelin-1 synthesis is also reduced by oestradiol. Progesterone modulates the effect of vasopressin on the uterine arteries in a dosedependent manner mechanism: progesterone is a vasodilator alone or combined with micromolar oestradiol but is a vasoconstrictor at lower doses ( 1 to $10 \mathrm{nM})$ [32].

Other factors are also involved in the regulation of vascular tone. Prostaglandins are auto/paracrine molecules derived from the metabolism of arachidonic acid and are thus either vasoconstrictive $\left(\mathrm{PGF}_{2 \alpha}, \mathrm{TXA}_{2}\right)$ via an increase in intracellular $\mathrm{Ca}^{++}$and protein kinase $\mathrm{C}$ (PKC) stimulation or vasodilatory $\left(\mathrm{PGE}_{2}, \mathrm{PGD}_{2}\right.$, $\mathrm{PGI}_{2}$ ) via a mechanism mediated by adenylate cyclase and cAMP formation. During a normal pregnancy, the ratio of prostacyclin to thromboxan increases progressively throughout the pregnancy. Prostacyclin $\left(\mathrm{PGI}_{2}\right)$ is produced by vascular ET and trophoblast cells and has vasodilatory effects (by relaxing vessels contracted by angiotensin II) and muscle-relaxing and antiaggregating effects. Thromboxan $\mathrm{A}_{2}$ is synthesized by the platelets and is a powerful vasoconstrictor which also promotes platelet aggregation and uterine contractility. During a normal pregnancy, the intravascular plugs formed by the EVCTs at the junction between the spiral arteries and the intervillous spaces inhibit membrane lipid peroxidation and diminish $\mathrm{TXA}_{2}$ levels [33]. Accordingly, the predominance of $\mathrm{PGI}_{2} \mathrm{~s}$ action compared with that of $\mathrm{TXA}_{2}$ (i.e. an increase in the $\mathrm{PGI}_{2} / \mathrm{TXA}_{2}$ ratio) causes vasodilation and decreases vascular resistance. The absence of these plugs in preeclampsia (caused by a defect in the EVCT vascular invasion) reduces the $\mathrm{PGI}_{2} / \mathrm{TXA}_{2}$ ratio (by decreasing $\mathrm{PGI}_{2}$ synthesis and increasing that of $\mathrm{TXA}_{2}$ ) and leads to (i) augmentation in the sensitivity of maternal vessels to angiotensin II, (ii) the absence of vasodilatation and (iii) the formation of localized microthromboses. Moreover, prostaglandin's vasomotor action can be modified by vascular alterations (fibrin deposits, endothelial lesions, etc.). A number of studies have revealed the presence of these vascular lesions in the placentas of $57 \%$ of women with intrauterine growth retardation (IUGR) and $74 \%$ of women with hypertension. The lesions thus further reduce perfusion of the intervillous spaces.

During pregnancy, adrenocorticotropic hormone $(\mathrm{ACTH})$ is present in both the maternal and foetal circulations [34]. It is a more powerful vasodilator (by a factor of 187) than $\mathrm{PGI}_{2}$. In pregnancies complicated by preeclampsia or IUGR, ACTH levels in the umbilical artery are elevated in response to stress and the hypoxemia that results from diminished blood flow. Corticotropin releasing factor (CRF) is a powerful 
vasodilator (50 times $\mathrm{PGI}_{2}$ ) whose levels are higher during pregnancies complicated by hypertension or IUGR [34] as a response to diminished placental vascular flow. However, endothelial alterations reduce corticotropins vasodilatory activity.

The vasomotor peptides, such as vaso-intestinal peptide (VIP), substance P (SP) and calcitonin generelated peptide (CGRP), synthesized by nerve fibres near the uterine vessels also help regulate uteroplacental blood flow. Vaso-intestinal peptide and SP exert a vasodilatory action on uterine vessels contracted by vasopressin (with no effect on basal parietal tension) and on villous vessels contracted by PGF2 $\alpha$ [14]. In humans, CGRP appears to be a growth factor for the endothelial cells of the umbilical vein and an angiogenic factor under ischaemic conditions [35]. Experiments in rats show that CGRP may inhibit the hypertension induced by administration of L-NAME (an analogue of L-arginine that inhibits NOS) and may reduce the foetal mortality associated with hypertensive syndromes. However, CGRP does not improve birth weight [36]; this is explained by the fact that CGRP does not cross the placenta and only corrects the NO deficit on the mother's side.

The beneficial effects of calcium supplementation in reducing the risk of preeclampsia suggests that calcium plays a role in this condition [37,38]. Compared with a normal pregnancy, the platelets of a woman with preeclampsia have a higher intracellular calcium level, which is probably related to an increase in voltage-dependent calcium channel activity. Pregnancy and elevated blood oestradiol levels affect these channels and reduce the concentration of intracellular calcium.

\section{The uterine immune balance}

In theory, an implanting embryo is a potential target for various types of immune attacks: standard cellmediated lysis, NK cell lysis and lysis by cytotoxic antibodies associated with complement. During pregnancy, the embryo is protected from these dangers by several factors: its own early antigenicity, secretion of cytokines and local immunosuppressors, intrinsic resistance to cell lysis and the MCP-DAF system. Two phases occur: an initial maternal immunological reaction against the allograft, followed by the development of allogenic tolerance.

The trophoblast cells in contact with maternal blood do not possess class I or II HLA antigens. HLA $\mathrm{G}$ is expressed solely in the EVCTs at the foetal-maternal interface (where no other class I or II antigens are found). This particular pattern of HLA G expression plays a role in tolerance of the semi-allogenic graft constituted by pregnancy; the NK response is inhibited because the trophoblast cells are disguised as self cells.
Cytokines may be secreted by leukocytes having infiltrated into the decidua or may be synthesized by endometrial or trophoblast cells.

Cytokines involved in the inflammatory reaction: IL-1 is detected in the decidua and IL-1 receptors are found on endometrial epithelial cells. The embryo also secretes IL-1, suggesting that it controls its own development via interferon (IFN) - a trophic factor for the trophoblast and for which the embryo has receptors. Interleukin 1 appears to stimulate the production of IFN, IL-6 and E2 prostaglandin ( $\left.\mathrm{PGE}_{2}\right)$ and enable the expression of class II HLA antigens (HLA-DR) in the uterus.

Cytokines involved in trophoblast development: CSF 1, GM-CSF and G-CSF are secreted in large quantities in the decidua and cognate receptors are expressed by the early trophoblast. CSF 1 favours the growth of the trophoblast, whereas GM-CSF plays a direct role in the trophoblast's attachment and growth and in embryonic survival. The latter factor is secreted by $\mathrm{CD} 56^{+}$large granular lymphocytes (LGLs) present in the decidua. The G-CSF receptors required for implantation are found in the trophoblast and the decidua.

Immunosuppressive cytokines: IL-6 inhibits expression of IL-2 receptors, which would otherwise promote the proliferation of cytotoxic cells (T lymphocytes and NK cells), B lymphocytes and the development of antibody-dependent cell-mediated cytotoxicity. Interleukin-10 plays a key role in preventing embryo resorption by antagonising IFN $\gamma$ and tumour necrosis factor alpha (TNF $\alpha$ ).

Progesterone is also involved in maintaining the semi-allograft because of its local anti-inflammatory activity; it can inhibit phagocytosis and lymphocyte proliferation in the uterus, either directly (by blocking $\mathrm{CD}^{+} \mathrm{T}$ lymphocyte activity and proliferation induced by IL-1) or indirectly (by inducing the release of two immunosuppressor factors by the lymphocytes). These are T-suppressor-induced factor (TSIF) - antibodies against which can abort a pregnancy in mice - and progesterone-induced blocking factor (PIBF), which blocks the lysis of embryo fibroblasts by NK cells and inhibits mixed lymphocyte reactions by preventing TNF $\alpha$ secretion by cytotoxic cells ( $\mathrm{T}$ lymphocytes, NK cells, etc.). Progesterone also has an immunosuppressive action which works in synergy with the prostaglandins $\mathrm{E}$ (PGE) and inhibits $\mathrm{T}$ lymphocyte proliferation.

This anti-complement activity is caused by the embryo's expression of anti-complement molecules: membrane complement protein (MCP, also known as CD 46), protectin (CD 59, which stops complement from binding to the constant region of the antibody chain) and DAF (CD 55, which accelerates complement destruction). These molecules are expressed on 
the membranes of gametes, fertilized eggs and blastocysts and thus transform any cytotoxic antibody into a blocking antibody - a sub-class of facilitating antibodies. Hence, a humorally-mediated abortion is theoretically possible if these molecules are expressed weakly, incorrectly or not at all or are expressed at the wrong stage or in the wrong location.

\section{The uterine environment}

\section{Hydrosalpynx}

Analysis of the literature reveals that the hydrosalpynx has an impact on embryo implantation. In fact, in a personal meta-analysis of 7 studies between $\mathrm{X}$ and $\mathrm{Y}$ featuring 2321 embryos transferred with hydrosalpynx and 9526 without, the implantation and pregnancy rates per cycle differed significantly according to the presence and absence of hydrosalpinx, with implantation rates of $6.6 \%$ and $11.5 \%$, respectively, and pregnancy rates of $19.7 \%$ versus $30.3 \%$, respectively. The physiopathology of this interaction involves an increase in the endometrium's production of antiimplantation cytokines (TNF $\alpha$, IL-2 and IL-12) under the effect of the chronic endometritis following on from hydrosalpynx.

\section{Uterine contractions}

The impact of myometrial uterine contractions (UCs) during embryo transfer has been clearly demonstrated by Fanchin et al. [39] via image analysis of digitized ultrasound scans. The contractions are inversely proportional to the progesterone level at the time of transfer, since this hormone is known to have muscle-relaxant properties. When there were fewer than 3 UCs per minute, a mean progesterone level of $111 \mathrm{ng} / \mathrm{ml}$ led to implantation and pregnancy rates of $21 \%$ and $53 \%$, respectively. With more than 5 UCs per minute, the mean progesterone level was only $68 \mathrm{ng} / \mathrm{ml}$ and implantation and pregnancy rates of respectively $4 \%$ and $14 \%$ were recorded. This notion again confirms the requirement for satisfactory progesterone impregnation of the uterus, in order to achieve not only adequate endometrial maturation but also establishment of the hormone's muscle-relaxant effect on the myometrium.

\section{Cases of preeclampsia}

The placentas of women with preeclampsia express lower levels of MMP-9, HLA G and $\alpha 1 \beta 1$ than those of women with normal pregnancies; the integrin $\alpha 6 \beta 4$ level is similar and that of $\alpha 5 \beta 1$ is higher [40]. Moreover, neither the switch from E-cadherin to VE-cadherin nor VCAM-1 and PECAM-1 production occur [41]. These phenomena attest to loss of the cytotro- phoblasts' capacity for deep invasion. Invasive EVCTs dedifferentiate into syncytium (giant cells) that lack penetrating power; the increase in giant cells thus expresses this initial impulse [42]. A related finding is the higher frequency of preeclampsia and intrauterine growth restriction in nulliparas (75\% of cases): this may be associated with the fact that the arteries colonized during a first pregnancy can be more easily invaded during subsequent pregnancies. The decidual NK cells may explain why subsequent invasion is facilitated, since they can be thought of as storing the "endometrial memory" of paternal antibodies. Preeclampsia was similar in nulliparas and in multiparas who had changed partners $(3.2 \%$ and $3 \%$, respectively) but less frequent for multiparas with the same partner $(1.9 \%)$. A similar finding is noted in pregnancies after oocyte or sperm donation or a long period of barrier contraceptive use. Accordingly, we should consider preeclampsia to be a disease of primipaternity rather than primigravidity.

In, the second trophoblast invasion either does not occur or is incomplete because of the lack of intravascular plugs. This translates into the persistence of uterine vasoconstriction; blood intake into the intervillous spaces is diminished and foetal growth retardation ensues. The downstream consequence of this vasoconstriction is hypoxia [43], with an increase in lipid peroxidation and in the $\mathrm{TXA}_{2} / \mathrm{PGI}_{2}$ ratios [44], both of which accentuate vasoconstriction and platelet aggregation. Thromboses and disseminated fibrin deposits are usually found in the preeclampsia placenta [45]. Moreover, downstream hypoxia increases ET-1 production and diminishes that of $\mathrm{NO}-\mathrm{a}$ phenomenon that is also related to the reduced mechanical force exerted on the artery wall.

Preeclampsia is thus characterised by (i) an increase in systemic vascular resistance and vascular reactivity and (ii) a change in the distribution of pelvic blood flow, which precedes the onset of hypertension. All of these factors evidence the dysregulation of the normal vasomotor factors during pregnancy. The increase in the vasoconstrictor/vasodilator ratio is proportional to the severity of the preeclampsia, and NO metabolite levels also diminish. Rats treated with LNAME develop hypertension and proteinuria and the growth of their foetuses is retarded. Hence, NO donors may have therapeutic value because they reduce blood pressure, inhibit platelet activation and improve uterine and foetal haemodynamics [31].

Accordingly, it is appropriate to prescribe a preventive treatment with aspirin in pregnancies where the women have poor obstetrics histories of previous early severe preeclampsia or severe intrauterine growth retardation defined as $<3$ rd percentile [46]. It appears necessary to begin the treatment as early as possible: from 13 weeks or even earlier (depending on the 
pathophysiologic bases explained above) when the indication is associated with the obstetrical history. This early prescription will have as its goal to limit, but not to prevent, the cascade of biological events in the mother (increase of renin, angiotensin, and aldosterone) that follow the increase in vascular resistance and the obstetrical complications that can result from it. This treatment should be continued until a term of 35 weeks. In some indications, this treatment can begin even before conception (for autoimmune diseases such as antiphospholipid syndrome), combined with heparin or corticosteroid therapy. Although trials have been performed with varying doses of aspirin (from 50 to $150 \mathrm{mg}$ ), the optimal dose does not seem to have been established. Nonetheless, the initial dose usually prescribed is from $100-150 \mathrm{mg}$ per day.

Lastly, chronic hypoxia (resulting from a placentation defect) can induce the transcription of certain genes, including that of ET-1 (a vasoconstrictor), angiotensin conversion enzyme (a hypertensive agent), plasminogen activator [47] (stimulating formation of active TGF $\beta$, which inhibits the EVCT invasion) and cyclooxygenase-1 (COX-1, an enzyme involved in prostaglandin production).

These observations and the notion of a sequence of preeclampsia/early repeated abortions in the same patients suggest that these two diseases may be on a continuum.

During preeclampsia, trophoblast expression of HLA $G$ falls at the mRNA level, in proportion to the reduction in the number of trophoblast cells observed in this disease.

As normal pregnancy progresses, macrophage and $\mathrm{T}$ lymphocyte counts remain constant, whereas the number of LGL falls. The LGLs have a particular NK phenotype and play a role in graft immunity. Furthermore, HLA G can inhibit LGL NK activity. One role of these uterine myeloid cells is to produce cytokines. A successful pregnancy requires a dominant Th2 system (humoral-mediated immunity by cytokines). During preeclampsia, this relationship is inversed $(\mathrm{Th} 1>\mathrm{Th} 2)$, thereby increasing the rate of destruction of trophoblast cells $(\mathrm{Th} 1=$ cell-mediated immunity). In addition, anomalies in PGE2 and TGF $\beta$ production have been observed during preeclampsia.

Hence, preeclampsia is characterised by generalized endothelial cell dysfunction and is linked to several factors: fatty acids, lipoproteins, lipid peroxide, $\mathrm{TNF} \alpha$, fibronectin decay products and syncytiotrophoblast microvillous fragments. All these various factors are generated by a generalized, intravascular, inflammatory response which is present during pregnancy but exacerbated in preeclampsia [48]. During inflammation, levels of leukocyte adhesion proteins in the vascular system rise, due to premature stimulation by first thrombin and histamine and then, in the hours that follow, by IL1 or TNF $\alpha$. Vascular permeability then increases the cellular chemotaxis with phagocytosis.

\section{References}

[ 1] Giudice LC, Saleh W. Growth factors in reproduction. Trends Endocrinol Metab. 1995;6:60-69.

[2] Tourgeman DE, Slater CC, Stanczyk FZ, Paulson RJ. Endocrine and clinical effects of micronized estradiol administered vaginally and orally. Fertil Steril. 2001;75:200-202.

[3] Battaglia C, Regnani G, Marsella T, Facchinetti F, Volpe A, Venturoli S, Flamigni C. Adjuvant L-arginine treatment in controlled ovarian hyperstimulation: a double-blind, randomized study. Hum Reprod. 2002;17:659-665.

[4] Sher G, Fisch JD. Vaginal sildenafil (Viagra): a preliminary report of a novel method to improve uterine artery blood flow and endometrial development in patients undergoing IVF. Hum Reprod. 2000;15:806-809.

[ 5] Fanchin R, Righini C, Ayoubi JM, Olivennes F, de Ziegler D, Frydman R. New look at endometrial echogenicity: objective computer-assisted measurement predict endometrial receptivity in in vitro fertilization-embryo transfer. Fertil Steril. 2000;74:274-281.

[ 6] Noyes RW, Hertig AT, Rock J. Dating the endometrial biopsy. Am J Obstet Gynecol. 1975;122:262-263.

[7] Tabibzadeh S, Babaknia A. The signals and molecular pathways involved in implantation, a symbiotic interaction between blastocyst and endometrium involving adhesion and tissue invasion. Hum Reprod. 1995;10:1579-1602.

[ 8] Lessey BA, Castelbaum AJ, Buck CA, Lei Y, Yowell CW, Sun J. Further characterization of endometrial integrins during the menstrual cycle and in pregnancy. Fertil Steril. 1994;62:497506.

[ 9] Lessey BA, Castelbaum AJ, Sawin SJ, Buck CA, Shinnar R, Wilkins B. Abberant integrin expression in the endometrium of women with endometriosis. J Clin Endocrinol Metab. 1994;79:643-649.

[10] Creus M, Balasch J, Ordi J, Fabregues F, Casamitjana R, Quinto L, Coutifaris C, Vanrell JA. Integrin expression in normal and out-of-phase endometria. Hum Reprod. 1998;13:3460-3468.

[11] Damsky CH, Librach C, Lim KH, Fitzgerald ML, McMaster MT, Janatpour M, Zhou Y, Logan SK, Fisher SJ. Integrin switching regulates normal trophoblast invasion. Development. 1994;120:3657-3666.

[12] Pijnenborg R. Establishment of uteroplacental circulation. Reprod Nutr Develop. 1988;28:1581-1586.

[13] Meekins JW, Luckas MJM, Pijnenborg R, Mc Fadyen IR. Histological study of decidual spiral arteries and the presence of maternal erythrocytes in the intervillous space during the first trimester of normal human pregnancy. Placenta. 1997; 18:459-464.

[14] Hansen V, Maigaard S, Allen J, Forman A. Effects of vasoactive intestinal polypeptide and substance $P$ on human intramyometrial arteries and stem villous arteries in term pregnancy. Placenta. 1988;9:501-506.

[15] Ignarro LJ. Biosynthesis and metabolism of endotheliumderived nitric oxide. Ann Rev Pharmacol Toxicol. 1990;30:535-569.

[16] Poranen AK, Ekblad U, Uotila P, Ahotupa M. Lipid peroxidation and antioxidants in normal and pre-eclamptic pregnancies. Placenta 1996;17:401-405.

[17] Jaffe R, Dorgan A, Abramowicz JS. Color Doppler imaging of uteroplacental circulation in the first trimester: value in prediction pregnancy failure or complication. $A J R$. 1995; 164:1255-1258. 
[18] Jauniaux E, Watson AL, Hempstock J, Bao YP, Skepper JN, Burton GJ. Onset of maternal arterial blood flow and placental oxidative stress. A possible factor in human early pregnancy failure. Am J Pathol. 2000;157:2111-2122.

[19] Kam EP, Gardner L, Loke YW, King A. The rôle of trophoblast in the physiological change in decidual spiral arteries. Hum Reprod. 1999;14:2131-2138.

[20] Lin S, Shimizu I, Suehara N, Nakayama M, Aono T. Uterine artery doppler velocimetry in relation to trophoblast migration into the myometrium of the placental bed. Obstet Gynecol. 1995;85:760-765.

[21] Vicovac L, Aplin JD. Epithelial-mesenchymal transition during trophoblast differentiation. Acta Anat. 1996;156:202-216.

[22] Aplin JD. Hypoxia and human placental development. J Clin Invest. 2000;105:559-560.

[23] Goffin F, Malassine A, Evain-Brion D, Munaut C, Frankenne F, Fridman V, Dubois M, Uzan S, Merviel P, Foidart JM. Evidence of a limited contribution of feto-maternal interactions to trophoblast differentiation along the invasive pathway. Tissue Antigens. 2003;62:104-116.

[24] Kupferminc MJ, Eldor A, Steinman N, Many A, Bar-Am A, Jaffa A, Fait G, Lessing JB. Increased frequency of genetic thrombophilia in women with complications of pregnancy. $N$ Engl J Med. 1999;340:9-13.

[25] O'Shaughnessy KM, Fu B, Ferraro F, Lewis I, Downing S, Morris NH. Factor V Leiden and thermolabile methylenetetrahydrofolate reductase gene variants in an East Anglian preeclampsia cohort. Hypertension. 1999;33:1338-1341.

[26] Rigo J, Nagy B, Fintor L, Tanyi J, Beke A, Karadi I. Maternal and neonatal outcome of preeclamptic pregnancies: the potential roles of factor V Leiden mutation and 5,10 methylenetetrahydrofolate reductase. Hypertens Pregnancy 2000;19:163172.

[27] Myatt L. Current topic: control of vascular resistance in the human placenta. Placenta 1992;13:329-341.

[28] Mac Mahon LP, Redman CWG, Firth JD. Expression of the three endothelin genes and plasma levels of endothelin in preeclamptic and normal gestations. Clinical Science 1993; 85:417-424.

[29] Mac Queen J, Kingdom JC, Connell JM, Whittle MJ. Fetal endothelin levels and placental endothelin receptors in intrauterine growth retardation. Obstet Gynecol. 1993;82: 992-998.

[30] Jovanovic A, Grbovic L, Tilic I. Predominant role for nitric oxide in the relaxation induced by acetyl-choline in human uterine artery. Hum Reprod. 1994;9:387-393.

[31] Moncada S, Higgs EA. Molecular mechanisms and therapeutic strategies related to nitric oxide. FASEB J. 1995;9:13191330.

[32] Mabry White M, Zamudio S, Stevens T. Estrogen, Progesterone, and vascular reactivity: potential cellular mecanisms. Endoc Rev. 1997;16:739-751.

[33] Davidge ST, Hubel CA, Mc Laughlin MK. Lipid peroxidation increases arterial cyclooxygenase activity during pregnancy. Am J Obstet Gynecol. 1994;170:215-222.
[34] Clifton VL, Read MA, Boura ALA, Robinson PJ, Smith R. Adrenocorticotropin causes vasodilatation in the human fetalplacenta circulation. J Clin Endoc Metab. 1996;81:14061410.

[35] Carter TL, Park P, Pratt K. Effects of CGRPa on the migration of endothelial cells of human origin. FASEB J. 1993;7:125.

[36] Yallampalli C, Dong YL, Wimalawansa SJ. Calcitonin generelated peptide reverses the hypertension and significantly decreases the fetal mortality in pre-eclampsia rats induced by $\mathrm{NG}^{\mathrm{G}}$-nitro-L-Arginine methyl ester. Hum Reprod. 1996;11: 895-899.

[37] Knight KB, Keith RE. Calcium supplementation on normotensive and hypertensive pregnant women. Am J Clin Nutr. 1992;55:891-895.

[38] Sanchez-Ramos L, Briones DK, Kaunitz AM, Delvalle GO, Gaudier FL, Walker CD. Prevention of pregnancy-induced hypertension by calcium supplementation in angiotensin IIsensitive patients. Obstet Gynecol. 1994;84:349-353.

[39] Fanchin R, Righini C, Olivennes F, Taylor S, de Ziegler D, Frydman R. Uterine contractions at the time of embryo transfer alter pregnancy rates after in-vitro fertilization. Hum Reprod. 1998;13:1968-1974.

[40] Merviel P, Challier JC, Carbillon L, Foidart JM, Uzan S. The role of integrins in human embryo implantation. Fetal Diagn Ther. 2001;16:364-371.

[41] Zhou Y, Damsky CH, Chiu K, Roberts JM, Fisher SJ. Preeclampsia is associated with abnormal expression of adhesion molecules by invasive cytotrophoblasts. J Clin Invest. 1993;91:950-960.

[42] Zhou Y, Damsky CH, Fisher SJ. Preeclampsia is associated with failure of human cytotrophoblast to mimic a vascular adhesion phenotype. One cause of defective endovascular invasion in this syndrome? J Clin Invest. 1997;99:2152-2164.

[43] Genbacev O, Joslin R, Damsky CH, Polliotti BM, Fisher SJ. Hypoxia alters early gestation human cytotrophoblast differentiation/invasion in vitro and models the placental defects that occur in preeclampsia. J Clin Invest. 1996;97:540-550.

[44] Walsh SW. Preeclampsia: an imbalance in placental prostacyclin and thromboxane production. Am J Obstet Gynecol. 1985;152:335-340.

[45] Tsukimori K, Maeda H, Shingu M, Koyanagi T, Nobunaga M, Nakano H. The possible role of endothelial cells in hypertensive disorders during pregnancy. Obstet Gynecol. 1992; 80:229-233.

[46] Uzan S, Merviel P, Beaufils M, Breart G, Salat-Baroux J. Aspirin during pregnancy. Indications and modalities of prescription after the publication of the later trials. Presse Med. 1996;25:31-36

[47] Estelles A, Gilabert J, Grancha S, Yamamoto K, Thinnes T, Espana F, Aznar J, Loskutoff DJ. Abnormal expression of type 1 plasminogen activator inhibitor and tissue factor in severe preeclampsia. Thromb Haemost. 1998;79:500-508.

[48] Redman CWG, Sacks GP, Sargent IL. Preeclampsia: an excessive maternal inflammatory response to pregnancy. $\mathrm{Am}$ J Obstet Gynecol. 1999;180:499-506. 